\title{
Folding Simulations of an $\alpha$-Helical Hairpin Motif $\alpha$ t $\alpha$ with Residue-Specific Force Fields
}

\author{
Juan Zeng, ${ }^{\dagger}$ Fan Jiang, ${ }^{*}{ }^{\dagger}$ and Yun-Dong $\mathrm{Wu}^{*}, \dagger, \dagger$ \\ $†$ Laboratory of Computational Chemistry and Drug Design, Laboratory of Chemical Genomics, \\ Peking University Shenzhen Graduate School, Shenzhen, 518055, China \\ $\$$ College of Chemistry and Molecular Engineering, Peking University, Beijing, 100871, China
}

\section{Supporting Information}

Figure S1. The time evolutions of the overall order parameters from $300 \mathrm{~K}$ MD simulations of uncapped $\alpha$ t $\alpha$ using AMBER ff99SB, ff99SB-ILND and OPLS-AA/L force fields.

Figure S2. Initial structures for the REMD simulation using the RSFF1 force field.

Figure S3. Initial structures for the REMD simulation using the RSFF2 force field.

Figure S4. All continuous REMD trajectories projected on $Q$ (upper) or RMSD (lower) folding reaction coordinate.

Figure S5. The simulated distance distributions of seven inter-helix side-chain pairs compared with experimental NOE restraints.

Table S1. The ${ }^{3} J_{\mathrm{H \alpha HN}}-$ coupling constants from experiments and simulations.

Figure S6. The representative structures from $1 \mu$ s $300 \mathrm{~K}$ MD simulations of capped and uncapped at $\alpha$ using RSFF1, superimposed with its NMR structure.

Figure S7. One continuous trajectory with folding transition event from the RSFF1 simulation. 

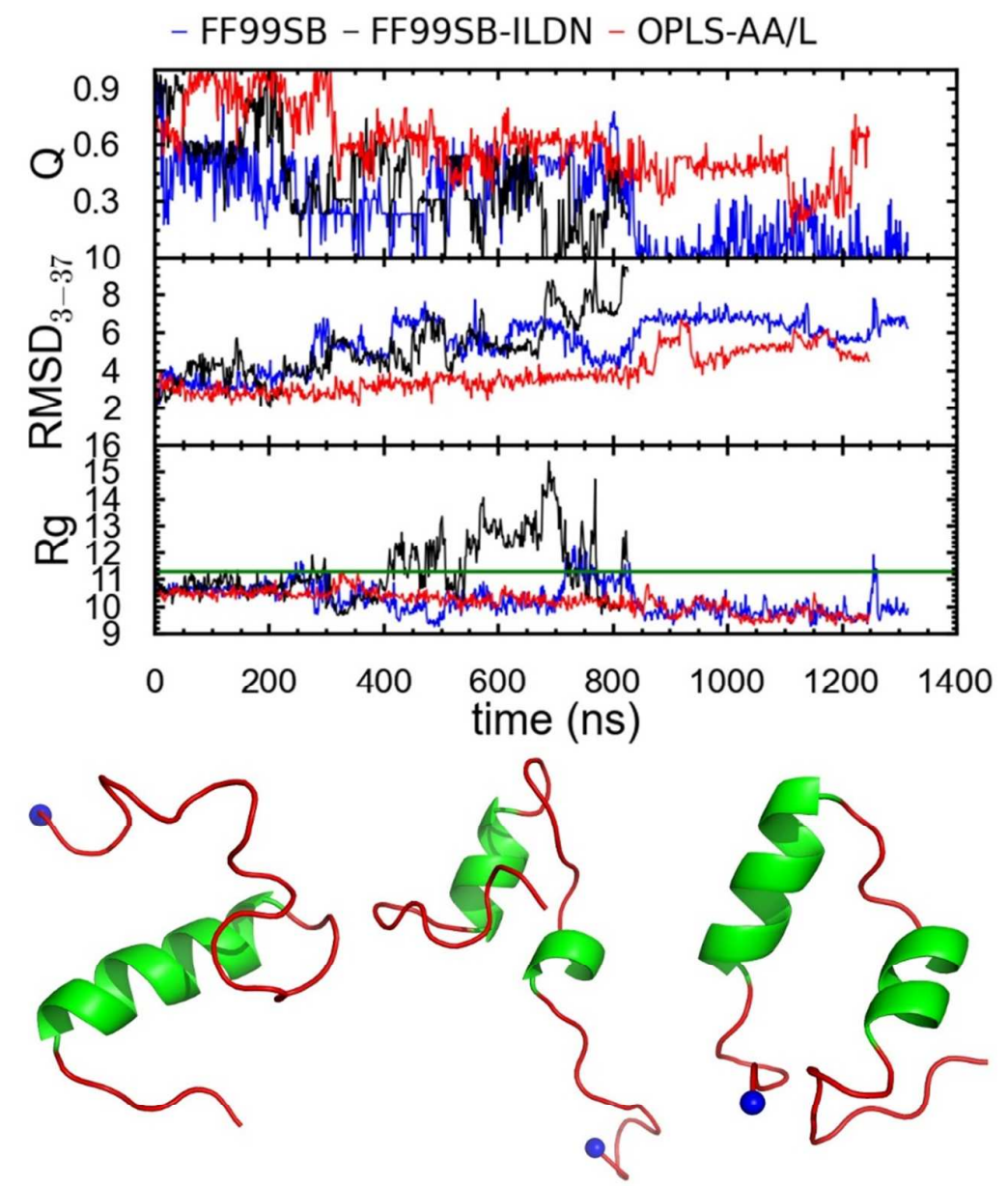

FF99SB $(0.83 \mu \mathrm{s})$

FF99SB-ILDN (1.2 $\mu \mathrm{s}) \quad$ OPLS-AA/L $(1.2 \mu \mathrm{s})$

Figure S1: The time evolutions of the fraction of native contacts $(Q)$, the $\mathrm{C} \alpha-\mathrm{RMSD}_{3-37}$ and the radius of gyration $(\mathrm{Rg})$ from $300 \mathrm{~K}$ MD simulations of uncapped $\alpha \mathrm{t} \alpha$ using AMBER ff99SB, ff99SB-ILND and OPLS-AA/L force fields. All three simulations were initiated from the first model in the NMR structure ensemble (1ABZ). The C $\alpha-\mathrm{RMSD}_{3-37}$ and $\mathrm{Rg}$ are in $\AA$, and the green line shows the $\mathrm{Rg}$ of the NMR structure. The structure at certain time (in parenthesis) is also shown for each simulation. 


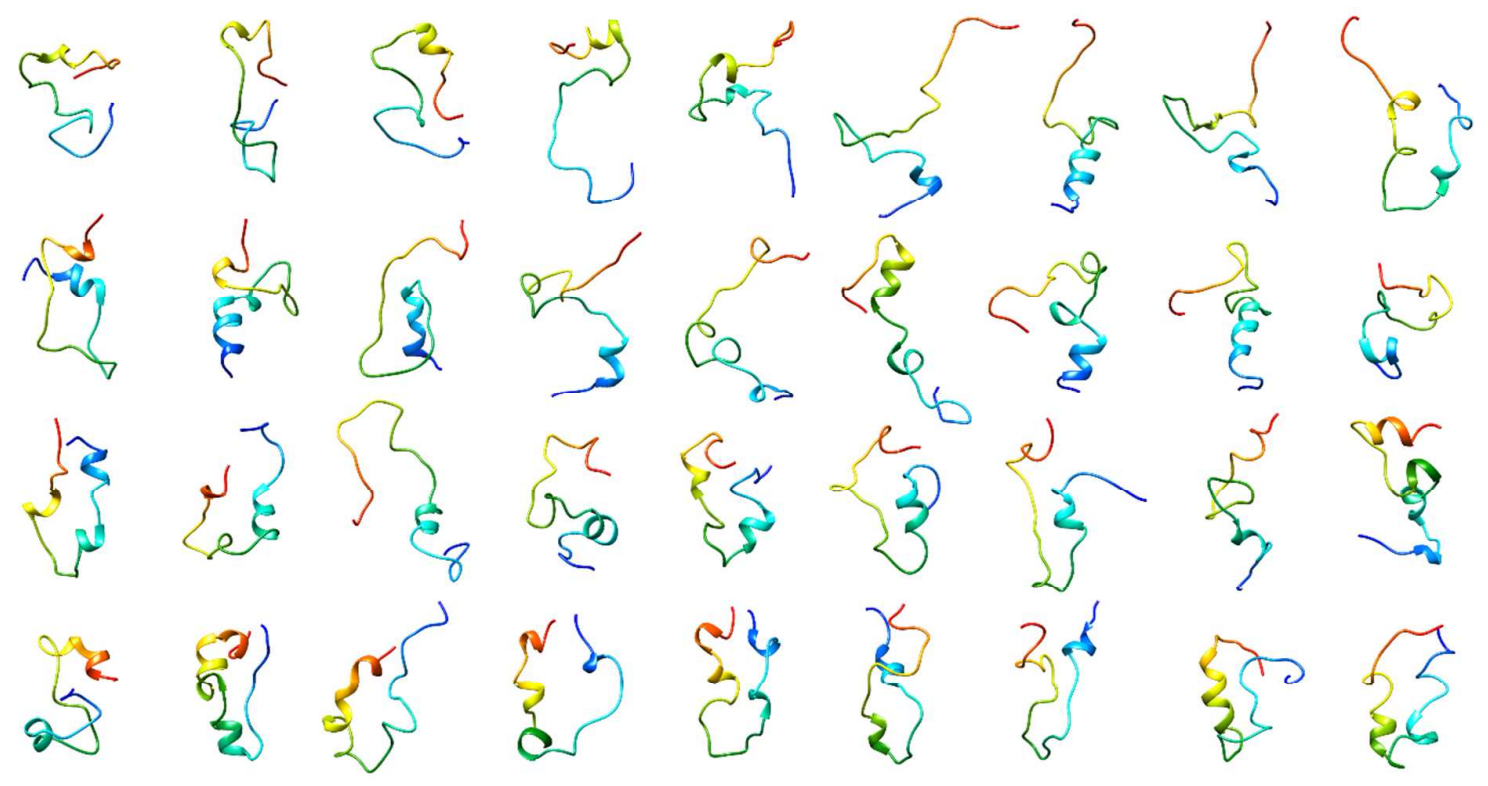

Figure S2. Initial structures for the REMD simulation using the RSFF1 force field.

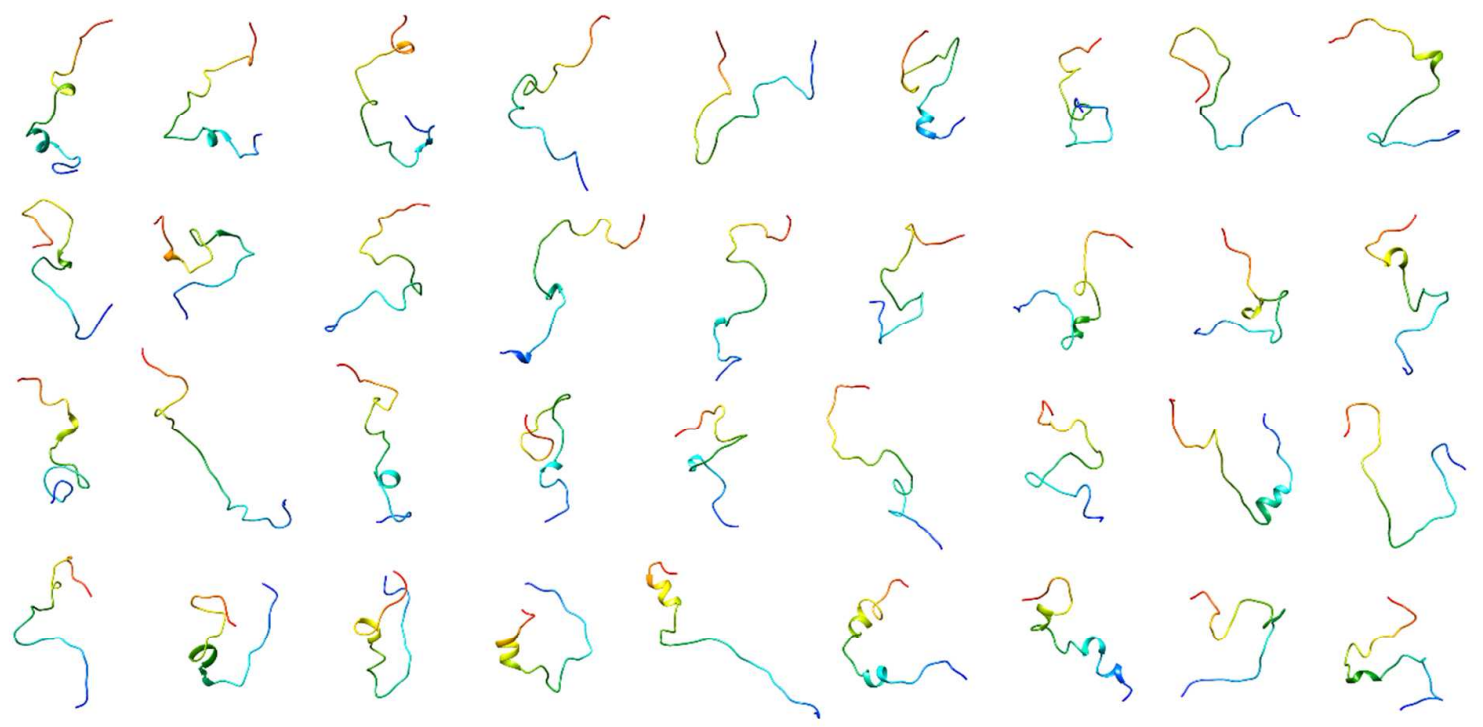

Figure S3. Initial structures for the REMD simulation using the RSFF2 force field. 

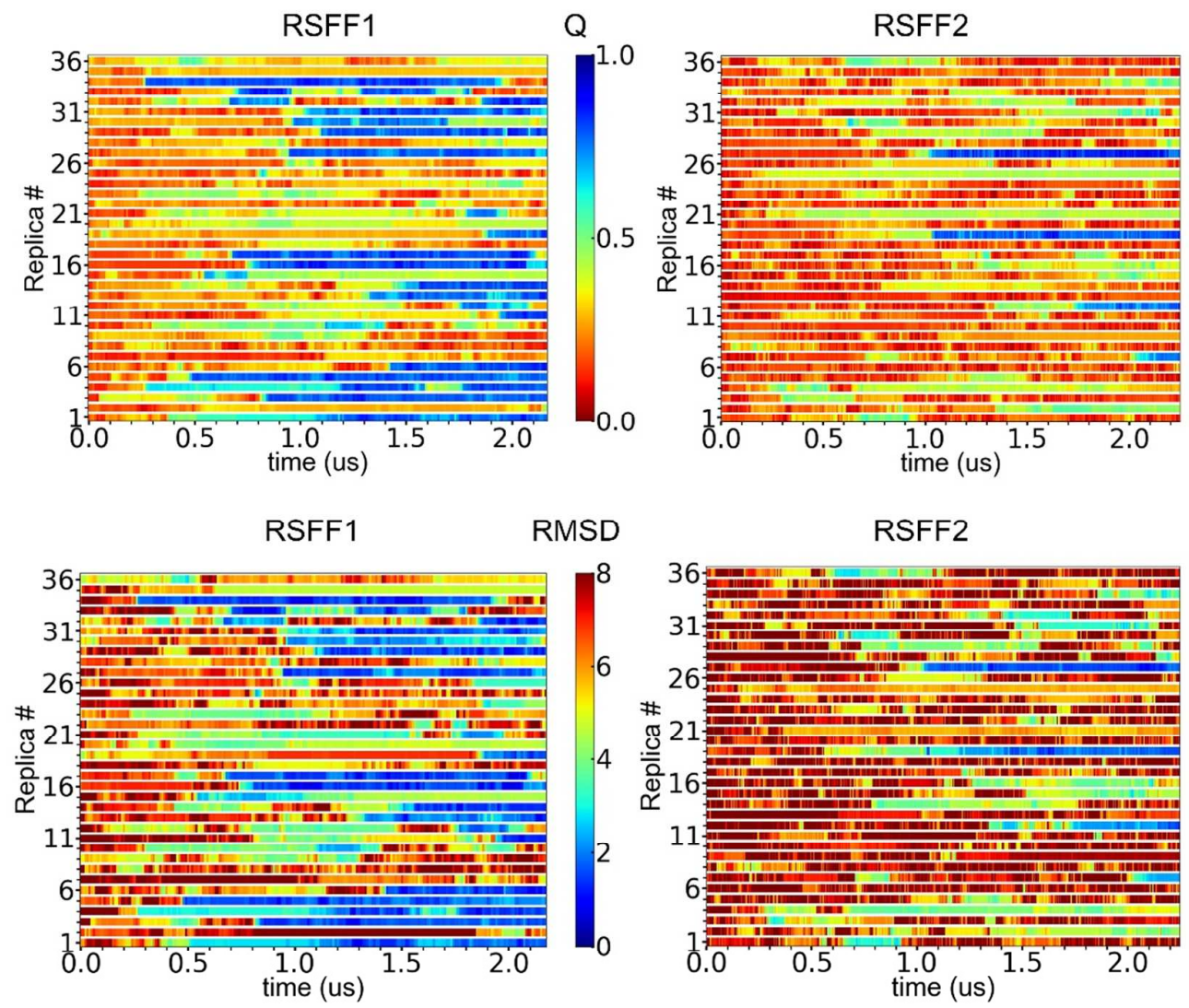

Figure S4. All continuous REMD trajectories projected on $Q$ (upper) or RMSD (lower) folding reaction coordinate. They were obtained by tracking every replica exchange in the REMD simulations, and values were averaged over $10 \mathrm{~ns}$ time blocks. Blue color indicates folded state. 

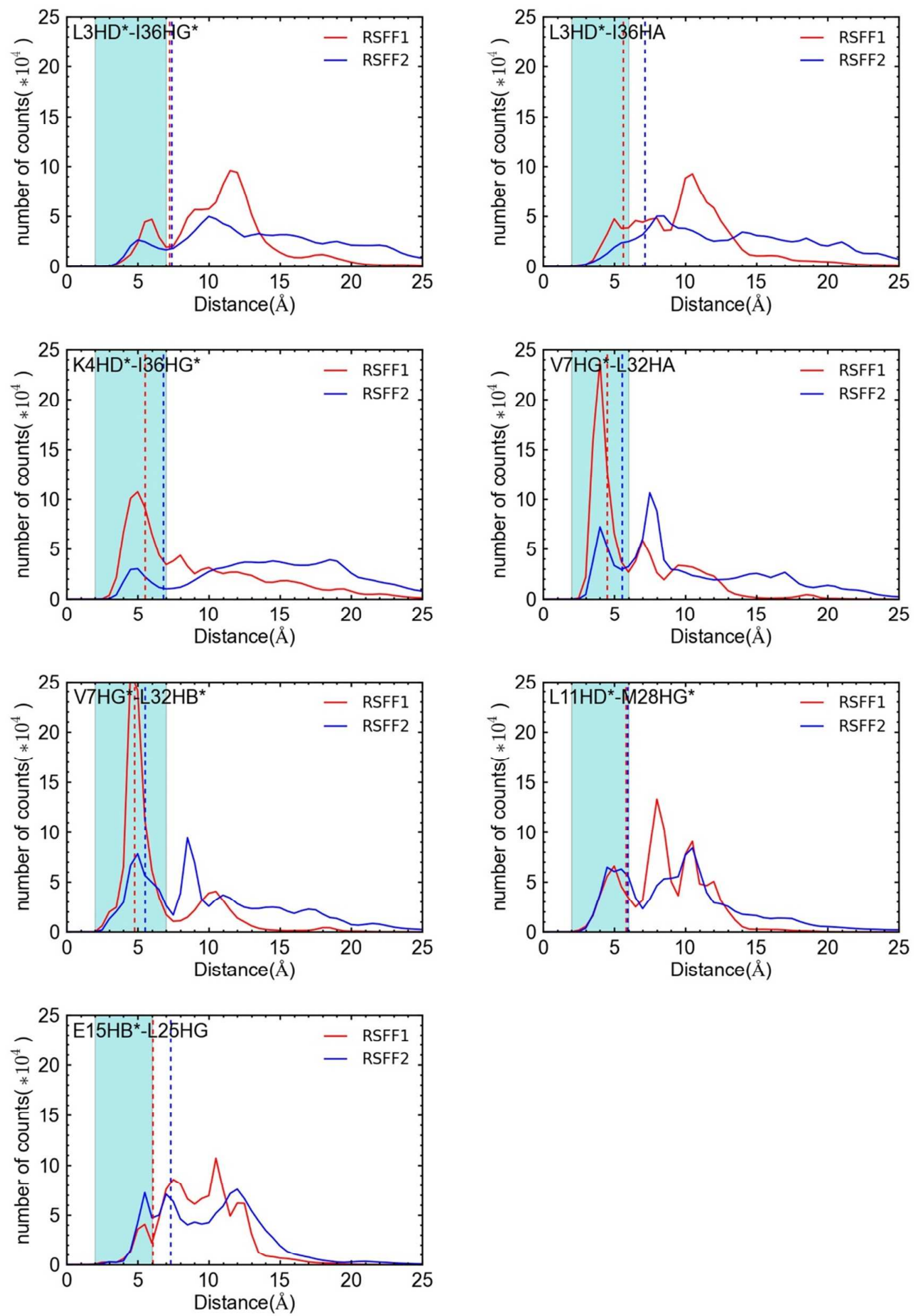

Figure S5: The simulated distance distributions of seven inter-helix side-chain pairs compared with experimental NOE restraints. The star represents that the calculation is based on the center of group. The shadow highlights the region between the lower and upper bounds used for NMR determination. The dashed lines are the $\left\langle\mathrm{r}^{-6}\right\rangle^{-1 / 6}$ average distances from the simulations. 
Table S1: The ${ }^{3} J_{\mathrm{H \alpha HN}}-$ coupling constants from experiment and simulations from RSFF1 and RSFF2 force field.

\begin{tabular}{cccc}
\hline Residue Index & Expt. & RSFF1 & RSFF2 \\
\hline W2 & $<6$ & 6.28 & 6.61 \\
L3 & $<6$ & 5.66 & 5.18 \\
K4 & $<6$ & 4.55 & 5.48 \\
A5 & & 3.65 & 4.48 \\
R6 & $<6$ & 4.83 & 5.06 \\
V7 & $<6$ & 4.61 & 5.12 \\
E8 & & 3.48 & 4.04 \\
Q9 & $<6$ & 3.74 & 4.46 \\
E10 & & 4.32 & 4.42 \\
L11 & $<6$ & 3.67 & 4.10 \\
Q12 & $<6$ & 3.51 & 4.13 \\
A13 & & 4.06 & 4.26 \\
L14 & $<6$ & 4.62 & 4.70 \\
E15 & & 4.76 & 4.77 \\
A16 & $<6$ & 5.08 & 4.91 \\
R17 & $>6$ & 6.48 & 6.06 \\
G18 & $>6$ & 5.13 & 5.52 \\
T19 & $>6$ & 6.60 & 6.28 \\
D20 & $>6$ & 6.50 & 5.94 \\
S21 & $>6$ & 6.35 & 5.99 \\
N22 & $>6$ & 4.73 & 5.46 \\
A23 & & 3.37 & 3.59 \\
E24 & $<6$ & 4.42 & 4.33 \\
L25 & $<6$ & 4.32 & 3.86 \\
R26 & & 3.39 & 3.63 \\
A27 & $<6$ & 4.00 & 4.09 \\
M28 & $<6$ & 4.22 & 4.39 \\
E29 & $<6$ & 3.50 & 3.67 \\
A30 & $<6$ & 3.96 & 3.84 \\
K31 & & 4.73 & 4.87 \\
L32 & $<6$ & 5.12 & 5.43 \\
K33 & $<6$ & 4.90 & 5.44 \\
A34 & $<6$ & 5.53 & 5.65 \\
E35 & $<6$ & 6.48 & 5.66 \\
I36 & $>6$ & 6.54 & 6.66 \\
Q37 & $<6$ & 7.12 & 6.61 \\
\hline & & &
\end{tabular}




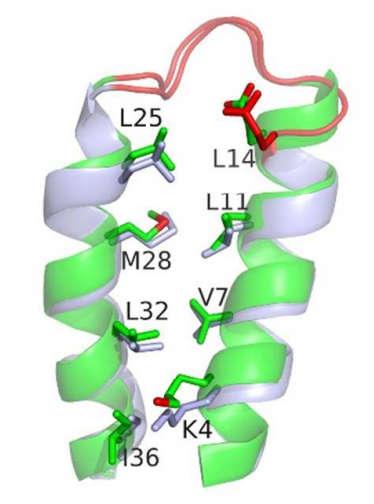

NMR vs RSFF1 (cap): $1.7 \AA$

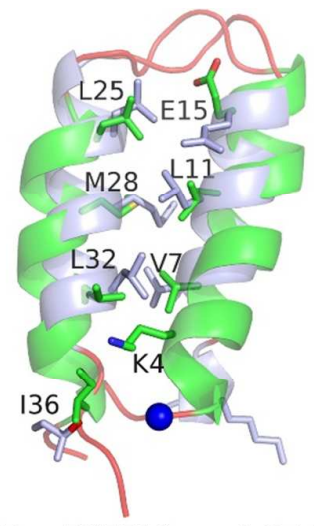

Figure S6. The representative structures (grey) from $1 \mu$ s $300 \mathrm{~K}$ MD simulations of capped and uncapped at $\alpha$ using RSFF1, superimposed with the NMR structure (green). The MD simulations were initiated from the first model of the NMR ensemble (1ABZ), and the RSFF1 force field was used. The C $\alpha-R M S D$ for residues 3 to $37\left(\mathrm{RMSD}_{3-37}\right)$ is also given for each simulated structure. Side-chains of key residues involving inter-helix association are labeled and shown in sticks. 

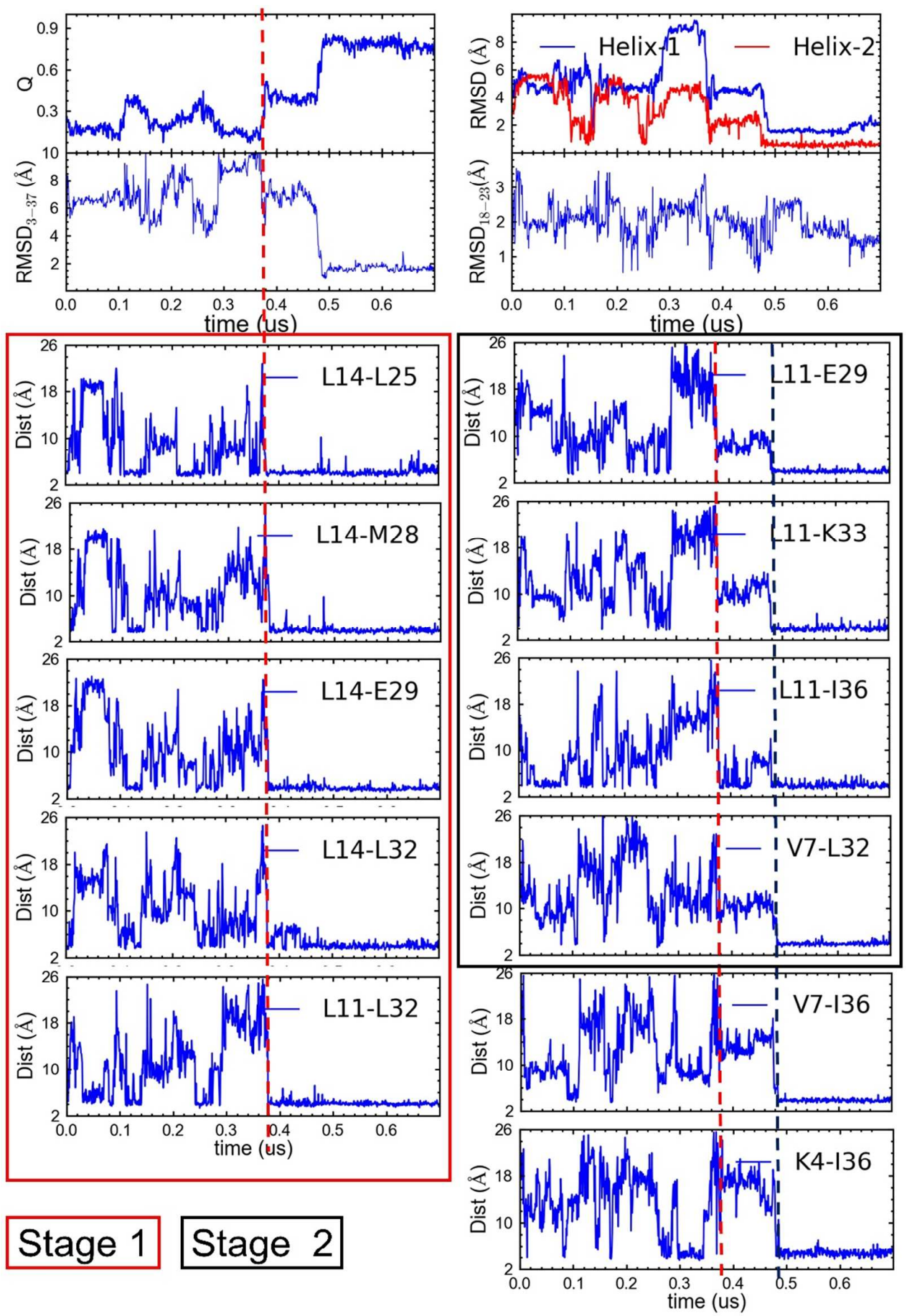

Figure S7. One continuous trajectory with folding transition event from the RSFF1 simulation. Three progress variables are traced: (i) the fraction of native contacts $(Q)$, (ii) $\mathrm{C} \alpha$-RMSD of residue 3 to 37, the helix-1, the helix-2, and the loop region, (iii) distances of eleven inter-helix side-chain pairs. 